\title{
Random sources for rectangular far fields
}

\author{
Olga Korotkova \\ Department of Physics, University of Miami, Coral Gables, Florida 33146, USA (korotkova@physics.miami.edu)
}

Received August 1, 2013; revised November 22, 2013; accepted November 24, 2013; posted November 25, 2013 (Doc. ID 195068); published December 19, 2013

A class of random sources producing far fields with rectangular intensity profiles is introduced by modeling the source degree of coherence with the help of two one-dimensional multi-Gaussian distributions. By changing the rms correlation widths along the $x$ and $y$ directions and the number of terms in the summation of the multi-Gaussian functions, the shapes and the edge sharpness of the beams can be adjusted. The results based on the derived analytical expression for the far-zone spectral density are supplemented by the computer simulations. @ 2013 Optical Society of America

OCIS codes: (030.1640) Coherence; (030.6600) Statistical optics; (260.0260) Physical optics.

http://dx.doi.org/10.1364/OL.39.000064

The variety of known models for the source correlation functions include Gaussian [1] $], J_{0}$-Bessel [2],$I_{0}$-Bessel [ㄹ] multi-Gaussian [ㄴ, $\underline{5}], \quad J_{0}$-Bessel-Gaussian, LaguerreGaussian [] ], cosine-Gaussian [7], and super-Gaussian (nonuniform correlations) [8]. All these models lead to far fields with circular symmetry being either Gaussian, flat, or ring shaped. In this Letter, we introduce a novel random source which produces far fields with flat square/rectangular intensity distributions. Such far fields may have any intensity distribution in the source plane but acquire the square/rectangular profile as the field passes to the far zone. The pattern then remains invariant on further propagation while enlarges due to diffraction.

The sources and fields of this Letter should not be confused with those considered in $[\underline{9}, 10]$ for which the source intensity has rectangular profiles and which change to Gaussian intensity profiles on propagation.

Suppose that a random beam-like field is generated by a planar source located in the plane $z=0$ and propagates along the positive $z$ direction. In the case when the source is of Schell-type, its second-order correlation properties can be characterized by the cross-spectral density function of the form [1]

$$
W^{(0)}\left(\rho_{1}^{\prime}, \rho_{2}^{\prime} ; \lambda\right)=\left[S^{(0)}\left(\rho_{1}^{\prime} ; \lambda\right) S^{(0)}\left(\rho_{2}^{\prime} ; \lambda\right)\right]^{1 / 2} \mu^{(0)}\left(\rho_{1}^{\prime}-\rho_{2}^{\prime} ; \lambda\right),
$$

where $\rho_{1}^{\prime}=\left(x_{1}^{\prime}, y_{1}^{\prime}\right)$ and $\rho_{2}^{\prime}=\left(x_{2}^{\prime}, y_{2}^{\prime}\right)$ are the twodimensional position vectors transverse to the direction of propagation, $S\left(\rho^{\prime} ; \lambda\right)$ is the spectral density, and $\mu^{(0)}\left(\rho_{1}^{\prime}, \rho_{2}^{\prime} ; \lambda\right)$ is the spectral degree of coherence. Let us now choose the spectral degree of coherence to be

$$
\begin{aligned}
\mu^{(0)}\left(\boldsymbol{\rho}_{1}^{\prime}, \boldsymbol{\rho}_{2}^{\prime} ; \lambda\right)= & \frac{1}{C^{2}} \sum_{m=1}^{M}\left(\begin{array}{l}
M \\
m
\end{array}\right) \frac{(-1)^{m-1}}{\sqrt{m}} \exp \left[-\frac{\left(x_{1}^{\prime}-x_{2}^{\prime}\right)^{2}}{2 m \delta_{x}^{2}(\lambda)}\right] \\
& \times \sum_{m=1}^{M}\left(\begin{array}{l}
M \\
m
\end{array}\right) \frac{(-1)^{m-1}}{\sqrt{m}} \exp \left[-\frac{\left(y_{1}^{\prime}-y_{2}^{\prime}\right)^{2}}{2 m \delta_{y}^{2}(\lambda)}\right],
\end{aligned}
$$

with normalization factor

$$
C=\sum_{m=1}^{M}\left(\begin{array}{l}
M \\
m
\end{array}\right) \frac{(-1)^{m-1}}{\sqrt{m}},
$$

where $\left(\begin{array}{l}M \\ m\end{array}\right)$ are the binomial coefficients, and $\delta_{x}(\lambda)$ and $\delta_{y}(\lambda)$ are the rms correlation widths along the $x$ and $y$ directions that can have quite arbitrary dependence on the wavelength. In what follows, the spectral dependence of all the quantities is omitted for brevity.

Figure 1 shows the absolute value of the degree of coherence $(\overline{2})$ for four selected sets of parameters: (a) and (b) $\delta_{x}(\lambda)=\delta_{y}(\lambda)=0.8 \mathrm{~mm}$; (c) and (d) $\delta_{x}(\lambda)=1.6 \mathrm{~mm}$, $\delta_{y}(\lambda)=0.56 \mathrm{~mm}$, (a) and (c) $M=1$; and (b) and (d) $M=40$.

In order to establish that the source (1)-(3) can be physically realizable (genuine) we employ the sufficient condition for the cross-spectral density function derived in [11]. According to this condition, the cross-spectral density (1) is genuine if it can be represented as superposition
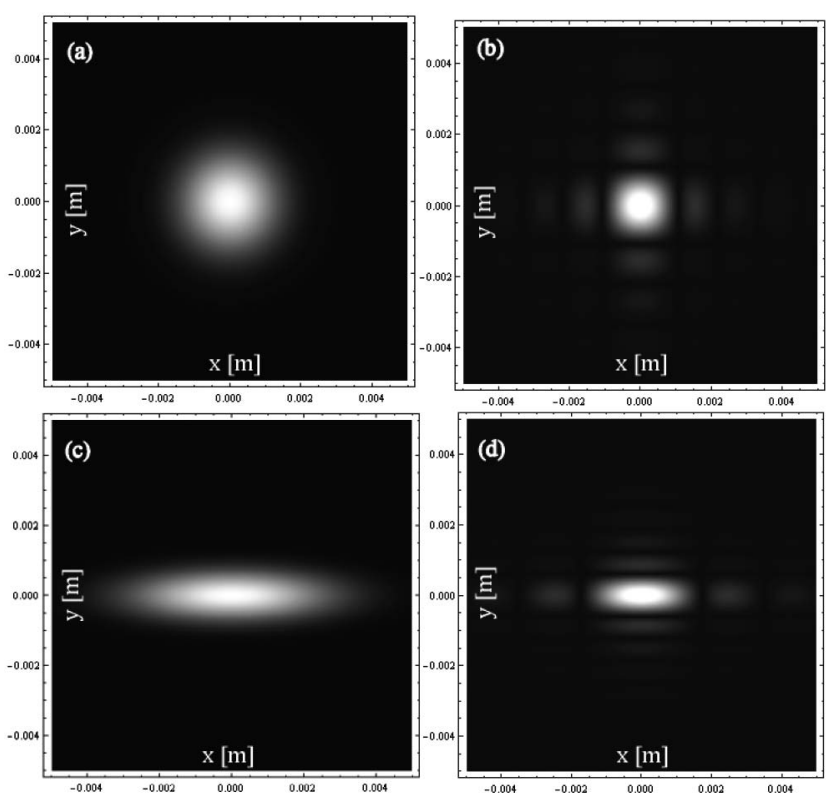

Fig. 1. Absolute value of the degree of coherence versus $x_{1}^{\prime}-x_{2}^{\prime}$ (horizontal) and $y_{1}^{\prime}-y_{2}^{\prime}$ (vertical). The horizontal and vertical scales extend from $-5 \mathrm{~mm}$ to $5 \mathrm{~mm}$. 


$$
\begin{aligned}
W^{(0)}\left(x_{1}^{\prime}, y_{1}^{\prime}, x_{2}^{\prime}, y_{2}^{\prime}\right)= & \iint p\left(v_{x}, v_{y}\right) H^{*}\left(x_{1}^{\prime}, y_{1}^{\prime}, v_{x}, v_{y}\right) \\
& \times H\left(x_{1}^{\prime}, y_{1}^{\prime}, v_{x}, v_{y}\right) \mathrm{d} v_{x} \mathrm{~d} v_{y}
\end{aligned}
$$

Here, $H$ is an arbitrary function, the asterisk stands for complex conjugate, and $p$ is a nonnegative, Fourier-transformable function. For the classic Schell-model correlations [1]

$$
H\left(x^{\prime}, y^{\prime}, v_{x}, v_{y}\right)=\tau\left(x^{\prime}, y^{\prime}\right) \exp \left[-2 \pi i\left(v_{x} x^{\prime}+v_{y} y^{\prime}\right)\right],
$$

where $\tau$ is the complex amplitude profile. The crossspectral density takes on the general form

$$
\begin{aligned}
W^{(0)}\left(x_{1}^{\prime}, y_{1}^{\prime}, x_{2}^{\prime}, y_{2}^{\prime}\right)= & \tau^{*}\left(x_{1}^{\prime}, y_{1}^{\prime}\right) \tau\left(x_{2}^{\prime}, y_{2}^{\prime}\right) \\
& \times \tilde{p}_{x}\left(x_{1}^{\prime}-x_{2}^{\prime}\right) \tilde{p}_{y}\left(y_{1}^{\prime}-y_{2}^{\prime}\right) .
\end{aligned}
$$

Here, the tilde denotes one-dimensional Fourier transform. In order to determine $p\left(v_{x}, v_{y}\right)$, it suffices to calculate the Fourier transform of Eq. (2)

$$
\begin{aligned}
p\left(v_{x}, v_{y}\right)= & \frac{\delta_{x} \delta_{y}}{C^{2}} \sum_{m=1}^{M}\left(\begin{array}{c}
M \\
m
\end{array}\right)(-1)^{m-1} \exp \left[-\frac{m \delta_{x}^{2} v_{x}^{2}}{2}\right] \\
& \times \sum_{m=1}^{M}\left(\begin{array}{l}
M \\
m
\end{array}\right)(-1)^{m-1} \exp \left[-\frac{m \delta_{y}^{2} v_{y}^{2}}{2}\right],
\end{aligned}
$$

or alternatively

$$
\begin{aligned}
p\left(v_{x}, v_{y}\right)= & \delta_{x} \delta_{y}\left\{1-\left(1-\exp \left[-\delta_{x}^{2} v_{x}^{2} / 2\right]\right)^{M}\right\} \\
& \times\left\{1-\left(1-\exp \left[-\delta_{y}^{2} v_{y}^{2} / 2\right]\right)^{M}\right\} / C^{2} .
\end{aligned}
$$

Since each of the two functions in the curly brackets is nonnegative for all the values of their arguments, the same is true for their product. Thus, the source with correlation (2) is physically realizable. Note that for large values of $M$ the degree of source coherence $\mu$ in Eq. (2) reduces to a "sinc" function. In fact, it follows directly from Eq. (8) that functions $p$ and $\mu$ are indeed the Fourier transform pair. Since for large values of $M p$ tends to form a 2D "rect-function," $\mu$ approaches the "sincfunction."

The amplitude profile function in Eq. (5) can be chosen at will, but in order to derive the analytic formulas we will set it to be Gaussian

$$
\tau\left(x^{\prime}, y^{\prime}\right)=\exp \left(-\frac{x^{\prime 2}+y^{\prime 2}}{4 \sigma^{2}}\right)
$$

with $\sigma$ as its rms source width. From Eqs. (2) and (9) we obtain for the cross-spectral density (1) the formula

$$
\begin{aligned}
W^{(0)}\left(\boldsymbol{\rho}_{1}^{\prime}, \boldsymbol{\rho}_{2}^{\prime} ; \lambda\right)= & \frac{1}{C^{2}} \exp \left(-\frac{x_{1}^{\prime 2}+x_{2}^{\prime 2}+y_{1}^{\prime 2}+y_{2}^{\prime 2}}{4 \sigma^{2}}\right) \\
& \times \sum_{m=1}^{M}\left(\begin{array}{c}
M \\
m
\end{array}\right) \frac{(-1)^{m-1}}{\sqrt{m}} \exp \left[-\frac{\left(x_{1}^{\prime}-x_{2}^{\prime}\right)^{2}}{2 m \delta_{x}^{2}(\lambda)}\right] \\
& \times \sum_{m=1}^{M}\left(\begin{array}{l}
M \\
m
\end{array}\right) \frac{(-1)^{m-1}}{\sqrt{m}} \exp \left[-\frac{\left(y_{1}^{\prime}-y_{2}^{\prime}\right)^{2}}{2 m \delta_{y}^{2}(\lambda)}\right] .
\end{aligned}
$$

We will term such a source the Rectangular Gaussian Schell model (RGSM) source.

The cross-spectral densities in the far field and in the source plane are related by a simple Fourier transform relation ([1] $]$ p. 240)

$$
\begin{aligned}
W^{(\infty)}\left(\mathbf{r}_{1}, \mathbf{r}_{2}\right)= & \frac{k^{2} \cos \theta_{1} \cos \theta_{2} \exp \left[i k\left(\mathbf{r}_{2}-\mathbf{r}_{1}\right)\right]}{(2 \pi)^{2} r_{1} r_{2}} \\
& \times \iint W^{(0)}\left(\boldsymbol{\rho}_{1}^{\prime}, \boldsymbol{\rho}_{2}^{\prime}\right) \exp \left[-i\left(\mathbf{f}_{1} \cdot \boldsymbol{\rho}_{1}^{\prime}\right.\right. \\
& \left.\left.+\mathbf{f}_{2} \cdot \boldsymbol{\rho}_{2}^{\prime}\right)\right] \mathrm{d}^{2} \boldsymbol{\rho}_{1}^{\prime} \mathrm{d}^{2} \boldsymbol{\rho}_{2}^{\prime},
\end{aligned}
$$

where $k=2 \pi / \lambda$ is the wave number, $\mathbf{r}_{1}=r_{1} \mathbf{s}_{1}, \mathbf{r}_{2}=r_{2} \mathbf{s}_{2}$ are the $3 \mathrm{D}$ vectors of points in the far zone with magnitudes $r_{1}=\left|\mathbf{r}_{1}\right|, \quad r_{2}=\left|\mathbf{r}_{2}\right|, \quad \mathbf{s}_{1}^{2}=\mathbf{s}_{2}^{2}=1, \quad \cos \theta_{1}=s_{1 z}$, $\cos \theta_{2}=s_{2 z}, \quad \mathbf{f}_{1}=-k \mathbf{s}_{1 \perp}, \quad \mathbf{f}_{2}=k \mathbf{s}_{2 \perp}, \quad s_{1 \perp}=\sin \theta_{1}$ and $s_{2 \perp}=\sin \theta_{2}$ (see Fig. 2). Since source (10) is separable in the Cartesian coordinate system, the $2 \mathrm{D}$ Fourier transform in Eq. (11) reduces to the product of two 1D transforms and one obtains the result

$$
\begin{aligned}
W^{(\infty)}\left(\mathbf{r}_{1}, \mathbf{r}_{2}\right)= & \frac{2 k^{2} \cos \theta_{1} \cos \theta_{2} \exp \left[i k r\left(\mathbf{s}_{2}-\mathbf{s}_{1}\right)\right]}{C^{2} r_{1} r_{2}} \\
& \times \sum_{m=1}^{M}\left(\begin{array}{c}
M \\
m
\end{array}\right) \frac{(-1)^{m-1} a_{m x}}{\sqrt{m} b_{m x}} \\
& \times \exp \left[-c_{m x}\left(s_{1 x}^{2}+s_{2 x}^{2}\right)-d_{m x}\left(s_{1 x}-s_{2 x}\right)^{2}\right] \\
& \times \sum_{m=1}^{M}\left(\begin{array}{c}
M \\
m
\end{array}\right) \frac{(-1)^{m-1} a_{m y}}{\sqrt{m} b_{m y}} \\
& \times \exp \left[-c_{m y}\left(s_{1 y}^{2}+s_{2 y}^{2}\right)-d_{m y}\left(s_{1 y}-s_{2 y}\right)^{2}\right],
\end{aligned}
$$

where

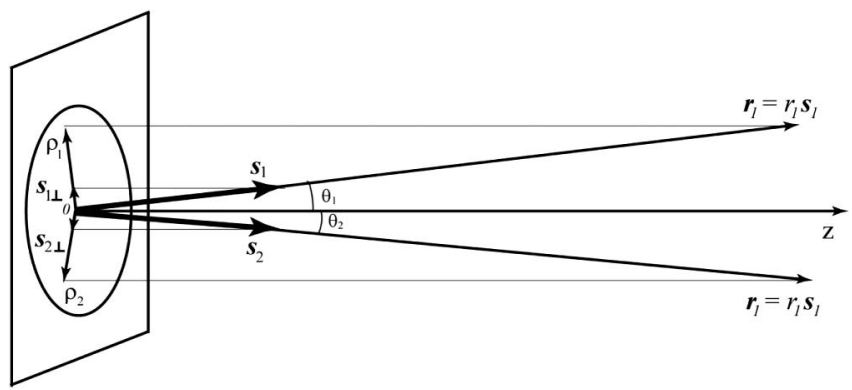

Fig. 2. Illustration of the notation related to the far field. 


$$
\begin{aligned}
& a_{m x}=\sigma \sqrt{\frac{2 m \delta_{x}^{2}+4 \sigma^{2}}{m \delta_{x}^{2}+4 \sigma^{2}}}, \quad b_{m x}=\sqrt{\frac{2}{m \delta_{x}^{2}}+\frac{1}{\sigma^{2}}}, \\
& c_{m x}=\frac{k^{2} \sigma^{2} m \delta_{x}^{2}}{m \delta_{x}^{2}+4 \sigma^{2}}, \quad d_{m x}=\frac{2 k^{2} \sigma^{4}}{m \delta_{x}^{2}+4 \sigma^{2}}, \\
& a_{m y}=\sigma \sqrt{\frac{2 m \delta_{y}^{2}+4 \sigma^{2}}{m \delta_{y}^{2}+4 \sigma^{2}}}, \quad b_{m y}=\sqrt{\frac{2}{m \delta_{y}^{2}}+\frac{1}{\sigma^{2}}}, \\
& c_{m y}=\frac{k^{2} \sigma^{2} m \delta_{y}^{2}}{m \delta_{y}^{2}+4 \sigma^{2}}, \quad d_{m y}=\frac{2 k^{2} \sigma^{4}}{m \delta_{y}^{2}+4 \sigma^{2}} .
\end{aligned}
$$

It follows from Eq. (12), with $\mathbf{r}_{1}=\mathbf{r}_{2}=\mathbf{r}$, that the spectral density distribution in the far field takes the form

$$
\begin{aligned}
S^{(\infty)}(r \mathbf{s}) & =\frac{2 k^{2} \cos ^{2} \theta}{C^{2} r^{2}} \sum_{m=1}^{M}\left(\begin{array}{c}
M \\
m
\end{array}\right) \frac{(-1)^{m-1} \sqrt{m} a_{m x}}{b_{m x}} \exp \left[-2 c_{m x} s_{x}^{2}\right] \\
& \times \sum_{m=1}^{M}\left(\begin{array}{l}
M \\
m
\end{array}\right) \frac{(-1)^{m-1} \sqrt{m} a_{m y}}{b_{m y}} \exp \left[-2 c_{m y} s_{y}^{2}\right] .
\end{aligned}
$$

Equation (14) implies that in order for the source (10) to generate a beam-like field its far-field spectral density must be negligible except for directions within a narrow solid angle about the $z$ axis [1]. This is so if

$$
\exp \left[-2 c_{x m} s_{x}^{2}\right] \approx 0, \quad \text { and } \quad \exp \left[-2 c_{y m} s_{y}^{2}\right] \approx 0,
$$

and hence if

$$
\frac{1}{4 \sigma^{2}}+\frac{1}{m \delta_{x}^{2}} \ll \frac{\pi^{2}}{\lambda^{2}} \quad \text { and } \quad \frac{1}{4 \sigma^{2}}+\frac{1}{m \delta_{y}^{2}} \ll \frac{\pi^{2}}{\lambda^{2}} .
$$

For values $m>1$, the inequalities (16) automatically hold if they do so for $m=1$. Thus, the beam conditions for the RGSM sources are

$$
\frac{1}{4 \sigma^{2}}+\frac{1}{\delta_{x}^{2}} \ll \frac{\pi^{2}}{\lambda^{2}} \quad \text { and } \quad \frac{1}{4 \sigma^{2}}+\frac{1}{\delta_{y}^{2}} \ll \frac{\pi^{2}}{\lambda^{2}} .
$$

Figure 3 shows the spectral density of the RGSM beams in the far zone as a function of position vectors $\mathbf{s}_{x}$ and $\mathbf{s}_{y}$ calculated from Eq. (14) for the same sets of source parameters as in Fig. 1. For sufficiently large values of index $M$ (for example 40), the spectral density acquires profiles with Cartesian symmetry.

The RGSM sources can be synthesized either by a method recently proposed in [12] or by reflecting a laser beam from the liquid crystal spatial light modulator (SLM) which acts as a random amplitude and/or phase plate with prescribed statistics $[\underline{13}, 14]$. For visual purposes, we will outline the latter approach by constructing a single realization of the amplitude screen and by obtaining the corresponding realization of the far field with the help of the 2D fast Fourier transform (FFT).

A 2D amplitude screen with the single-point Gaussian statistics and the two-point correlation function (2) can be constructed by convolving a $2 \mathrm{D}$ array of normally distributed random variables with the window function (2) [13]. The result of this procedure applied to the SLM
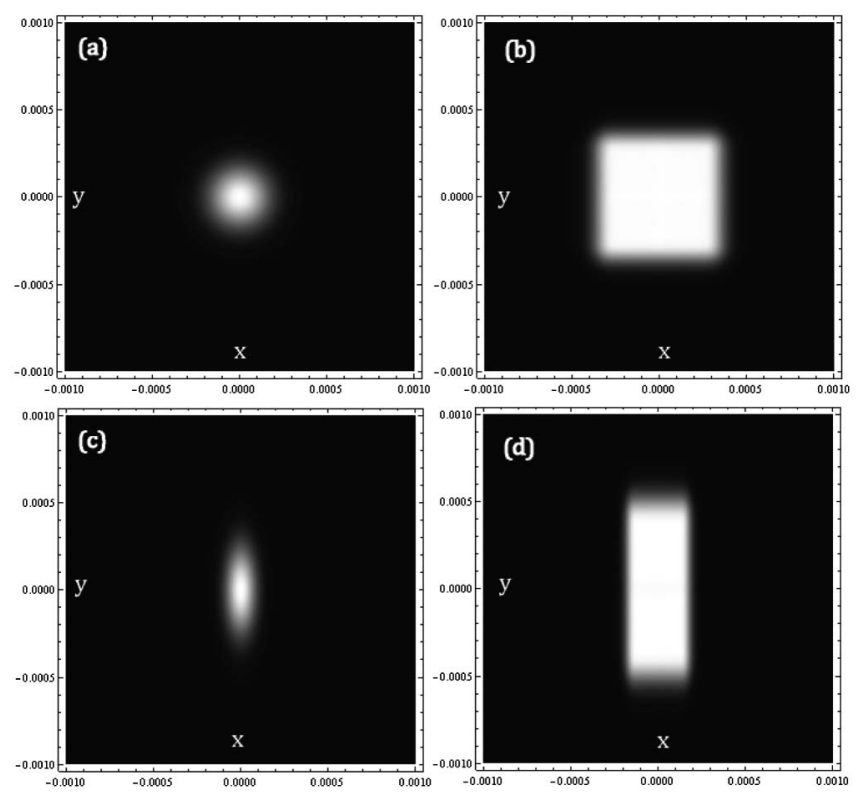

Fig. 3. Spectral density of the RGSM beams in the far zone of sources with parameters as in Fig. 1. The horizontal and vertical axes extend from $-1 \mathrm{~mm}$ to $1 \mathrm{~mm}$.

screen with generic $512 \times 512$ pixels is shown in Fig. $\underline{4}$ with the following parameters: (a) and (b) $\delta_{x}(\lambda)=$ $\delta_{y}(\lambda)=2$ pixels; (c) and (d) $\delta_{x}(\lambda)=1.4$ pixels, $\delta_{y}(\lambda)=$ 4 pixels; (a) and (c) $M=1$; and (b) and (d) $M=40$. The structure and the size of the speckles on the amplitude screens change as index $M$ grows and as the rms correlations $\delta_{x}(\lambda)$ and $\delta_{y}(\lambda)$ are modified.

In cases when $\sigma \ll \delta_{x}$ and $\sigma \ll \delta_{y}$, the source (10) can be regarded as quasi-homogeneous. Hence, on the basis of the well-known for such sources reciprocity relations [1], its far field spectral density is proportional to the source degree of coherence. Hence, on taking the FFT

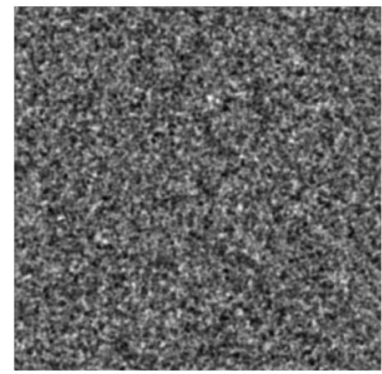

(a)

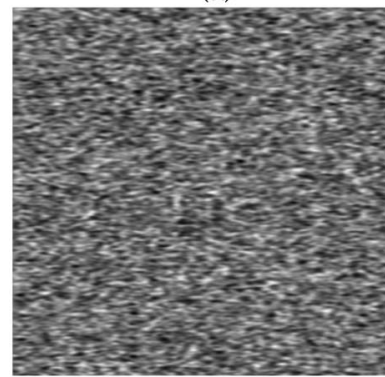

(c)

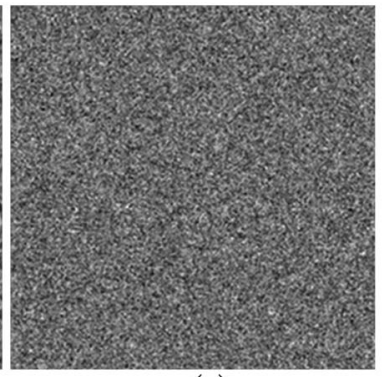

(b)

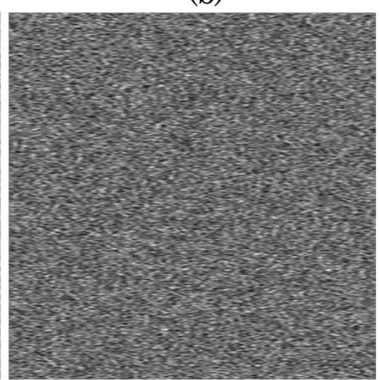

(d)
Fig. 4. Computer simulations of the random amplitude distribution for generation of sources with the degree of coherence (1), corresponding to Figs. $\underline{1}$ and $\underline{3}$. 


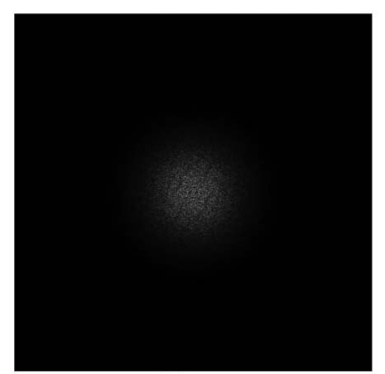

(a)

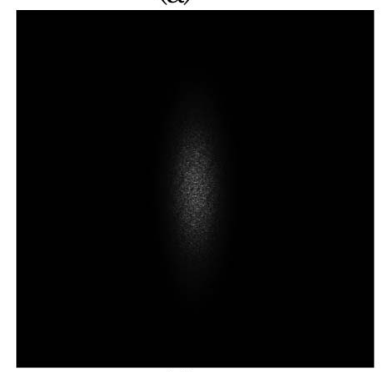

(c)

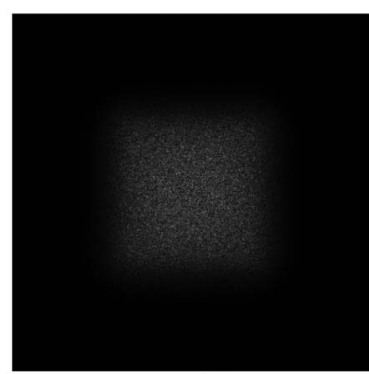

(b)

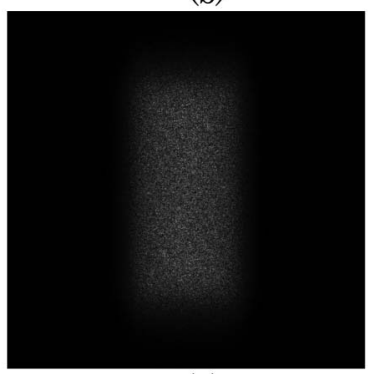

(d)
Fig. 5. Computer simulations of the far fields produced by sources with the degree of coherence (1). The source parameters are the same as in Fig. $\underline{4}$.

of the screens in Fig. $\underline{3}$ we can obtain the corresponding realizations of the far fields produced by the RGSM source (see Fig. 5). The comparison between Figs. $\underline{3}$ and 5 shows that the analytical result (14) and the simulation obviously produce similar results: averaging over sufficiently many distributions in Fig. 5 provides the sharp images of Fig. 3. It should be noted that even though the scales in Figs. 3 and 5 are different (the former is presented in mm and the later in pixels), the ratios of parameters were taken to be the same for each subfigure leading to the same geometrical profiles of the far fields.

In summary, random sources with properly chosen degree of coherence can produce far fields having intensity distributions with Cartesian symmetry, i.e., being either squares or rectangles. The suggested form of the degree of coherence (2) is a separable function in $x$ and $y$ directions, while both contributions are modeled as sums of weighted Gaussian functions with the same (leading to squares) or different (to rectangles) rms widths. We have illustrated that the function (2) describes the physically realizable random source, named the RGSM source. The analytic expression for far-field cross-spectral density for the RGSM source has been derived and the beam conditions have been established. Since the RGSM source is based on superposition of Gaussian functions, one benefits from its tractability on passage through optical systems and various media.

The summation index $M$ provides a convenient tool for adjusting the beam's edge sharpness. In this Letter, the index $M$ is assumed to be equal along the $x$ and $y$ directions. However, such an assumption can be readily removed leading to more flexibility in adjusting the sharpness of different edges. The far fields with Cartesian symmetry generated by the RGSM sources are shapeinvariant throughout the far zone. This is the unique feature that distinguishes them from all previously introduced rectangular flat-top beams whose intensity profiles are preserved only in close proximity to their sources.

The results of this work are of particular importance for a number of applications in which a field with rectangular symmetry must be generated from a source with arbitrary intensity distribution at a sufficiently large distance. Such applications can include free-space and atmospheric optical communications, imaging, and optical material surface processing.

The author is obliged to Prof. S. Avramov-Zamurovic and Dr. C. Nelson for sharing their insight into optical simulations and to Prof. E. Shchepakina for helpful discussions relating to this work. The research is sponsored by the US AFOSR (FA9550-12-1-0449) and the US ONR (N0018913P1226).

\section{References}

1. L. Mandel and E. Wolf, Optical Coherence and Quantum Optics (Cambridge University, 1997).

2. F. Gori, G. Guattari, and C. Padovani, Opt. Commun. 64, 311 (1987).

3. S. A. Ponomarenko, J. Opt. Soc. Am. A 18, 150 (2001).

4. S. Sahin and O. Korotkova, Opt. Lett. 37, 2970 (2012).

5. O. Korotkova, S. Sahin, and E. Shchepakina, J. Opt. Soc. Am. A 29, 2159 (2012).

6. Z. Mei and O. Korotkova, Opt. Lett. 38, 91 (2013).

7. Z. Mei and O. Korotkova, Opt. Lett. 38, 2578 (2013).

8. H. Lajunen and T. Saastamoinen, Opt. Lett. 36, 4104 (2011).

9. Y. Li, J. Mod. Opt. 50, 1957 (2003).

10. G. Wu, H. Guo, and D. Deng, Opt. Commun. 260, 687 (2006).

11. F. Gori and M. Santarsiero, Opt. Lett. 32, 3531 (2007).

12. F. Wang, X. Liu, Y. Yuan, and Y. Cai, Opt. Lett. 38, 1814 (2013).

13. T. Shirai, O. Korotkova, and E. Wolf, J. Opt. A 7, 232 (2005).

14. O. Korotkova, S. Avramov-Zamurovic, C. Nelson, and R. Malek-Madani, Proc. SPIE 8238, 82380J (2012). 